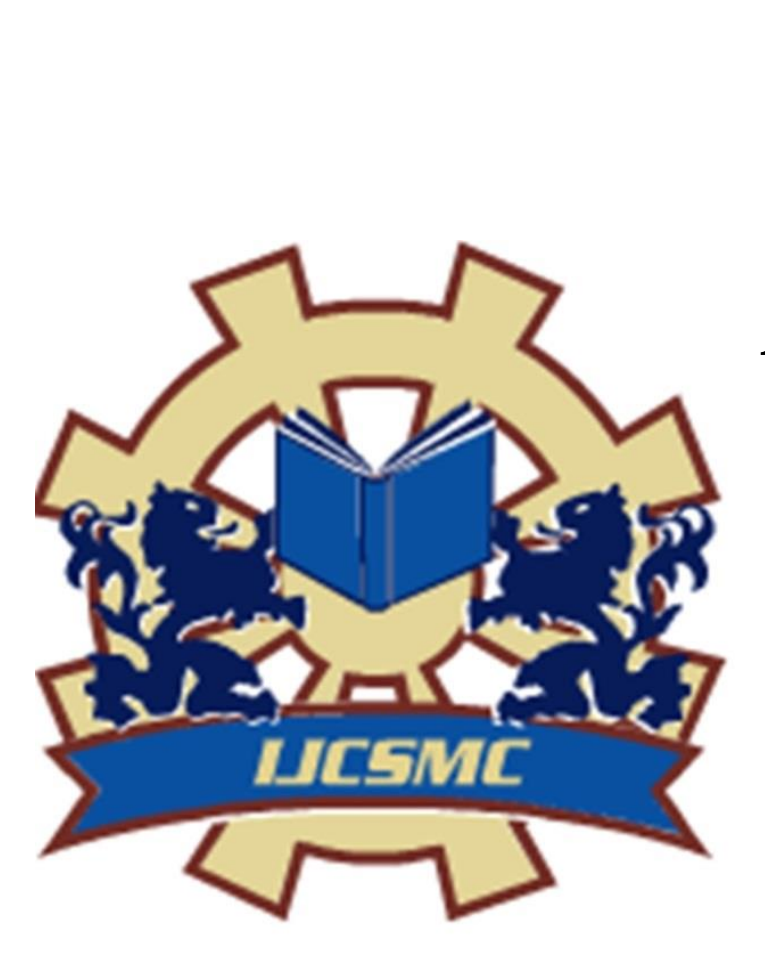

Available Online at www.ijesmc.com

International Journal of Computer Science and Mobile Computing

A Monthly Journal of Computer Science and Information Technology

ISSN 2320-088X
IMPACT FACTOR: 7.056

IJCSMC, Vol. 10, Issue. 10, October 2021, pg.83-88

Poster Presentation Article

\title{
YOLO Based Social Distancing Violation Detection
}

Raj Kumar Pal; Ranjan Keshri; Sandeep Verma; Subhomoy Chattopadhyay

DOI: 10.47760/ijesmc.2021.v10i10.009 


\section{YOLO Based Social Distancing Violation Detection}

Raj Kumar Pal, Ranjan Keshri, Sandeep Verma, Subhomoy Chattopadhyay



FINAL RESULT

The model is detecting only the human beings and calculating the distance between the nearest bounding box. This is how it is taking a decision between high risk and safe distances.

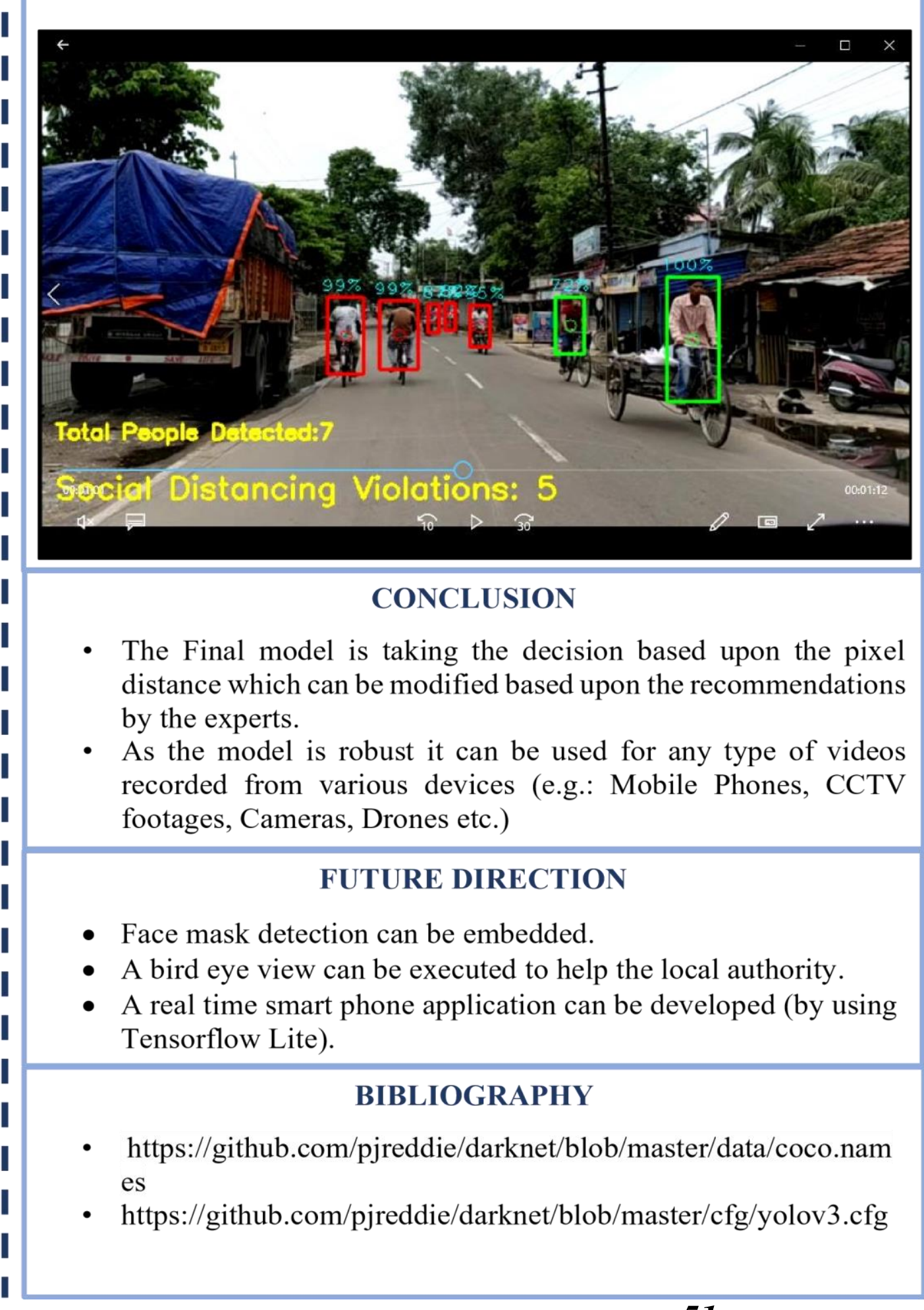

